\title{
What to Expect When No One's Expecting
}

\author{
by Jonathan V. Last \\ New York: Encounter Books 2013 \\ ISBN 978-1594036415 \\ Hardcover, \$28, 230 pp.
}

\author{
Reviewed by Maxwell Hartt \\ School of Planning, University of Waterloo
}

Decades ago, publications by the Club of Rome (1972) and Paul Ehrlich (1968) regarding overpopulation and its associated forthcoming global crisis made a significant academic and cultural impact across the globe. Since then, many of these theories have been debunked and the hysteria has died down; however, the concept of an overpopulated planet has remained in the social conscience. Jonathan V. Last's first book, What To Expect When No One's Expecting, aims to amend the widespread misinformation and misunderstanding, while voicing the actual threat to our future- shrinking populations.

In essence, this is a non-technical demographic book, geared to inform the general public. Although the primary focus is on America, Last details a wealth of international comparisons throughout the book. These comparisons are key to contextualizing the American fertility slump, the potential for demographic disaster and a variety of failed, successful, and proposed strategies to counter declining populations.

Last opens the book with an analysis of pet owners in America. Staggering statistics, such as the fact American pets outnumber American children by more than four to one, captivate the reader and begin sketching the foundation of the full story to come. Demographic terms (crude birth rate, completed fertility, total fertility rate, etc.) and the 'Golden Number' of replacement, 2.1, are defined simply and accessibly, to provide readers of all backgrounds a basic understanding of population estimates and forecasts. Last also makes a point of addressing a few loaded issues before delving fully into the content. First, he discusses Margaret Sanger and the concern of racism in demography. Second, he clarifies the difference between correlation and causation. And thirdly, he offers a "preemptive defense against readers who may take this book to be a criticism of the modern American woman."

What to Expect When No One's Expecting spans many topics but never treads far from its focal point-Americans are uninformed about a looming crisis of massive proportions. Specifically, readers can look forward to a historic examination of America's fertility trends, the specific costs of raising children in America, the notion and reality of "ideal fertility" the causes and solutions to America's fertility problem, and much more. What To Expect When No One's Expecting manages to cover a wide range of topics in significant detail without feeling forced or drawn out. Last's take on the roots of America's collapsing fertility - which include "the ubiquity of college, the delay of marriage, the birth-control pill, car seat laws, religious participation, the rise of the thousand dollar stroller, and Social Security" - are especially interesting, verging on controversial. Similarly, the solutions offered up in the final chapters are no less divisive, with the author suggesting more roads be built, a return to 1950s levels of religiosity, and earlier and more marriages, among others.

Just before these final solutions are proposed comes perhaps the most interesting chapter of the book, "Losing Battles (What Not to Do)." In this chapter, the author takes a thorough cross-national examination of various pro-natalist policies. From the Russian Motherhood Medal (given to women who mothered at least six children) to the Japanese law stating that any business with 300 or more employees must create a plan to raise 
the fertility rate of its workers, to a range of Singaporean strategies that included cash bonuses (up to $\$ 12,000$ ), tax credits, paid maternity leave, preferential housing, and school allotments, Last covers the topic thoroughly and captivatingly. These extreme comparisons help frame the seriousness of the issue.

Overall, this is a timely, important, and valuable contribution to general pop culture literature. Following in the same vein as Freakonomics, The Tipping Point, and Boom, Bust and Echo, Last looks to make a seemingly complex and analytical discussion more accessible to the general public. However, despite being well written, structured, and full of helpful analogies, What To Expect When No One's Expecting falls shy of the mark due to a few crucial shortcomings. Last's central argument of overlooked and oversimplified population trends cannot stand firmly, as he himself makes egregious simplifications and shallow criticisms of many important academic works. Additionally, Last's own conservative views ring throughout the book, leaving the reader with a distinct impression of subjectivity and bias, which unfortunately undermines the solid analytical arguments brought forth.

With these shortcomings in mind, this book is nevertheless an important step forward in the general public's acknowledgement and discussion of forthcoming demographic trends. By creating such a controversial piece, Last's work has the potential to spark debate and discussion in many forums outside of the demographic academe. So while it is doubtful that this book will inform academics or demographers (except perhaps with some of the international case studies or anecdotal evidence), it will bring much-needed and widespread focus to the issue of shrinking populations and fertility rates.

\section{References}

Ehrlich, P.R. 1968. The Population Bomb. New York: Ballantine Books.

Club of Rome. 1972. The Limits to Growth. New York: Universe Books. 